\title{
Working memory, math performance, and math anxiety
}

\author{
Mark H. Ashcraft And Jeremy A. Krause \\ University of Nevada, Las Vegas, Nevada
}

\begin{abstract}
The cognitive literature now shows how critically math performance depends on working memory, for any form of arithmetic and math that involves processes beyond simple memory retrieval. The psychometric literature is also very clear on the global consequences of mathematics anxiety. People who are highly math anxious avoid math: They avoid elective coursework in math, both in high school and college, they avoid college majors that emphasize math, and they avoid career paths that involve math. We go beyond these psychometric relationships to examine the cognitive consequences of math anxiety. We show how performance on a standardized math achievement test varies as a function of math anxiety, and that math anxiety compromises the functioning of working memory. High math anxiety works much like a dual task setting: Preoccupation with one's math fears and anxieties functions like a resource-demanding secondary task. We comment on developmental and educational factors related to math and working memory, and on factors that may contribute to the development of math anxiety.
\end{abstract}

This article provides an overview of the relationships among working memory, math performance, and math anxiety. We provide examples from the mathematical cognition literature to show: the critical role of working memory in performing arithmetic and math; the relationship between math performance and math anxiety, especially on standardized math achievement tests; and finally, the way that math anxiety compromises the functioning of working memory when people do arithmetic and math. We conclude with some predictions concerning the risk factors for math anxiety, and with some of the educational implications of this work. See Ashcraft and Ridley (2005) and Ashcraft, Krause, and Hopko (2007) for full-length treatments of these issues. Excellent summaries of the entire field of mathematical cognition can be found in Campbell (2005).

We begin with a statement concerning just one justification (of many) for this work. Math and science are in the headlines these days, with research-based reports about the relatively poor job American schools do in teaching math and science, and the depressingly substandard job many students are doing in mastering these topics. No one doubts the importance of math and science to the workforce in a technological society, or their importance in general to an educated populace. So there is a general, undeniable need for investigations about the learning and mastery of math. And from a disciplinary perspective, the rich complexity of math in all its facets suggests that it should be an interesting topic for cognitive psychology to address, and a critical one in any discussion of the relevance of cognitive psychology to education.

\section{Working Memory and Math Performance}

Considerable evidence has appeared in the past 10 to 15 years concerning the vital role that working memory plays in mathematical cognition. In LeFevre, DeStefano, Coleman, and Shanahan's (2005) view, the literature now supports a clear generalization concerning the important positive relationship between the complexity of arithmetic or math problems and the demand on working memory for problem solving. One aspect of this relationship involves the numerical values being manipulated, and one aspect examines the total number of steps required for problem solution. We take these in turn.

It is now clear that working memory is increasingly involved in problem solving as the numbers in an arithmetic or math problem (the "operands") grow larger. The benchmark effect in this area is the problem-size effect, the empirical result that response latencies and errors increase as the size of the operands increases: For example, $6+7$ or $9 \times 6$ will be answered more slowly and less accurately than $2+3$ or $4 \times 5$ (see Zbrodoff $\&$ Logan's 2005 review). Part of this effect, we have argued, is due to the structure of the mental representation of arithmetic facts in long-term memory, and the inverse relationship between problem size and problem frequency-for example, in textbooks (e.g., Hamann \& Ashcraft, 1986). That is, larger arithmetic problems simply occur less frequently, and hence are stored in memory at lower levels of strength (see Siegler \& Shrager, 1984, for a comparable approach); this is similar in most respects to the standard word-frequency effect found in language processing research. A second part of the effect, documented in the

M. H.Ashcraft, mark.ashcraft@unlv.edu 
past 10 years, is the increasing tendency for larger operand problems to be solved via some nonretrieval process, whether it be counting, reconstruction from known problems, or other strategies (see, e.g., Campbell \& Xue, 2001; LeFevre, Sadesky, \& Bisanz, 1996). Because nonretrieval processing is invariably found to be slower and more error prone than memory-based retrieval, the occurrence of strategy-based trials will slow down overall response latencies, especially for larger problems. Critically for the present discussion, strategy- or procedure-based performance will rely far more heavily on the resources of working memory in comparison with performance based on relatively automatic memory retrieval.

We illustrate this with a series of experiments reported in Seyler, Kirk, and Ashcraft (2003). In this work, we tested college adults on the "basic facts" of subtraction - that is, the inverses of the addition facts $0+0$ up to $9+9$. As shown in Figure 1, there was a gently increasing problemsize profile on response latency up to $10-n$ problems, but then a dramatic increase in reaction times (RTs) beginning with $11-n$ problems; error rates jumped from below $5 \%$ to the $10 \%-22 \%$ range at the same point. The dramatic change in the performance profiles suggested strongly that the larger subtraction problems were being solved via strategies. To test this possibility, we repeated the study, asking participants to answer the question "How did you solve the problem?" after each trial. The reported incidence of strategy use matched the RT and error profiles very closely; strategy use was reported an average of $3 \%$ of the time on small subtraction problems, but on $33 \%$ of the trials with large problems. On this evidence, simple

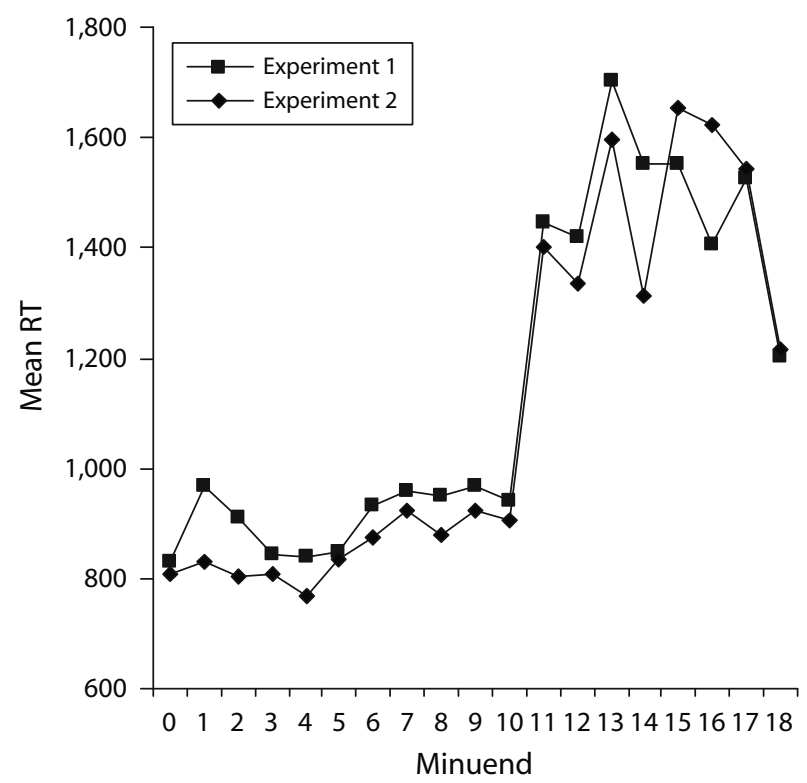

Figure 1. Mean reaction time (RT) to simple subtraction facts from $0-0$ to $18-9$ by minuend; for example, in $14-8$, the "minuend" is 14. From "Elementary subtraction," by D. J. Seyler, E. P. Kirk, \& M. H. Ashcraft, 2003, Journal of Experimental Psychology: Learning, Memory, \& Cognition, 29, p. 1341, Figure 1. Copyright 2003 American Psychological Association. Adapted with permission. subtraction is heavily reliant on strategy use, a pattern that should disadvantage participants if they are laboring under limited working memory resources.

To document this final prediction, we tested simple subtraction in a dual task setting: Participants held two, four, or six random letters in working memory while performing the subtraction; they then had to report the letters in serial order. The dual task led to a significant decrement in performance, as measured by accuracy of letter recall. Importantly, this decrement was especially pronounced for the large subtraction problems, those that relied heavily on strategies rather than on retrieval. And the pattern was exaggerated when participants' own working memory capacity was considered. There was substantially more interference with letter recall for the low-working-memoryspan participants (a 56\% error rate in the most difficult condition) than for the medium- or high-span groups (respectively, $46 \%$ and $31 \%$ error rates; Seyler et al., 2003, Figure 7). In short, there was an increasing cost of the dual task requirement for participants with lower working memory capacity. Simple subtraction, an arithmetic operation introduced routinely in second grade, has a substantial working memory component to it, especially because even adults continue to rely heavily on strategybased processing instead of memory retrieval. Such reliance disadvantages participants whose working memory is occupied by a secondary task, and also those whose working memory capacity is low.

The important point here is that strategy-based solutions are not just slower, but far more demanding on working memory, whereas memory retrieval is usually found to be a fast and relatively automatic process, with little or no demand on working memory resources. Reports consistent with this generalization are now common-for example, work showing the dramatic decline in latencies and working memory involvement as a function of practice on difficult math (Beilock, Kulp, Holt, \& Carr, 2004; Tronsky, 2005).

Similarly, the number of steps in a problem solution is generally strongly correlated with response times, and with the working memory resources necessary for correct solutions; this is roughly analogous to the increase in processing load with an increase in the number of clauses in a sentence, for instance. As an example, Hecht (2002) found that a concurrent articulatory task disrupted addition trials performed via counting far more than it did trials performed via retrieval (see comparable results in a test of sequential adding by Logie, Gilhooly, \& Wynn, 1994).

In our test of the relationship between number of steps and working memory (Ashcraft \& Kirk, 2001), we reasoned that the carry operation in addition should require additional working memory processing, because carrying adds yet another step to the processing sequence. We presented participants with addition problems ranging from basic addition facts up to two-column additions; half of all problems required a carry (e.g., $27+14$; see also Fürst \& Hitch, 2000). The results were clear cut (see Ashcraft \& Kirk, 2001, Figure 1). Carry problems were considerably slower than their noncarry counterparts, fully $1,200 \mathrm{msec}$ slower for the largest problems. Likewise, carry problems 
invariably had higher error rates (from $5.2 \%$ to $9.4 \%$ ) than their noncarry counterparts $(0.2 \%$ to $2.1 \%)$.

It appears that working memory processing is integral to arithmetic and mathematics performance whenever a procedure other than direct memory retrieval is operating. That is, when simple one-column addition or multiplication is being performed, the underlying mental process responsible is principally retrieval from memory, in which case working memory plays a minor role, at best. But when performance relies on algorithmic proceduressay, carrying —or when other reconstructive strategies are used, then working memory is crucial. Likewise, for multistep problems, there is an increasing reliance on working memory as the number of steps increases (see, e.g., Ayres, 2001), and at points in problem solving when the need for retaining intermediate goals and values is highest (see, e.g., Campbell \& Charness, 1990).

\section{Math Performance and Math Anxiety}

Serious research on math anxiety began to appear in the early 1970s, when a suitable objective instrument for measuring math anxiety became available. Since that time, scores of articles have appeared on the various psychometric properties of the original scale and its descendants, and on the relationships between math anxiety and a host of other characteristics. The best summary of this work remains the Hembree meta-analysis (1990), which, for the most part, is the source of the following correlations between math anxiety and various aspects of math performance.

The story told by the correlations is sad indeed. The higher one's math anxiety, the lower one's math learning, mastery, and motivation; for example, a math anxiety correlation of -.30 with high school grades, -.75 with enjoyment of math, -.64 with motivation to take more math or do well in math, and -.31 with the extent of high school math taken. The overall correlation between math anxiety and individuals' math achievement, as measured by standardized tests, is -.31 . Thus, highly math-anxious individuals get poorer grades in the math classes they take, show low motivation to take more (elective) math, and in fact do take less math. They clearly learn less math than their low-anxious counterparts.

These correlations mean, simply but importantly, that as math anxiety increases, math achievement declines. This seemingly inherent relationship between math anxiety and achievement poses a genuine interpretive quandary: Is lower performance on a math task due to math anxiety or to lower mastery and achievement in math? Fortunately, our work suggests a partial way out of the quandary. That is, we collected scores from some 80 undergraduates on a math-anxiety assessment and also on the Wide Range Achievement Test (WRAT), a standard math achievement test. The correlation between math anxiety and the composite WRAT score was -.35 , very close to the value in Hembree's (1990) meta-analysis. But we then rescored the WRAT performance, taking advantage of its line-byline increases in difficulty (e.g., whole-number addition in Line 1, multiplication of fractions in Line 5, solving for two unknowns in Line 8). When the test is scored in this fashion, the impact of math anxiety is much clearer; see Figure 2. Simple accuracy is at ceiling for all groups on the initial lines of the test, suggesting no evidence of lower achievement per se for math-anxious individuals on the whole-number arithmetic taught in elementary school (i.e., even high-anxious individuals can answer wholenumber problems correctly). Likewise, when we gave untimed paper-and-pencil tests of our whole-number arithmetic stimuli, we found no math-anxiety differences on accuracy, even though these same stimuli generated online anxiety effects in an RT task (Faust, Ashcraft, \& Fleck, 1996). But group performance on the WRAT does start to diverge around Line 4 or 5: On the most difficult line of the test, the high-anxious group averages fewer than one in five problems correct. Thus, the lower achievement of math-anxious individuals seems limited to more difficult math, the math taught at or after late elementary school.

Note a second point as well. Scores on such achievement tests probably underestimate levels of math achievement among high-anxious participants. When students take math tests, especially high-stakes math achievement tests, it is very likely that their online anxiety reaction is disrupting their performance. In agreement with this, Hembree (1990) noted that for groups who undergo effective interventions for their math anxiety (cognitive behavioral interventions), math achievement scores approach those in the normal range. Given that the interventions do not provide any instruction or practice in math, it follows that previously low achievement-test results could be at least partially explained by an online anxiety reaction that depressed preintervention scores.

The negative relationship between math anxiety and achievement is not universal across all forms of arithmetic and math, and not universal across all testing situations. Lab testing at the simpler levels of arithmetic need not worry about a confounding relationship between achieve-

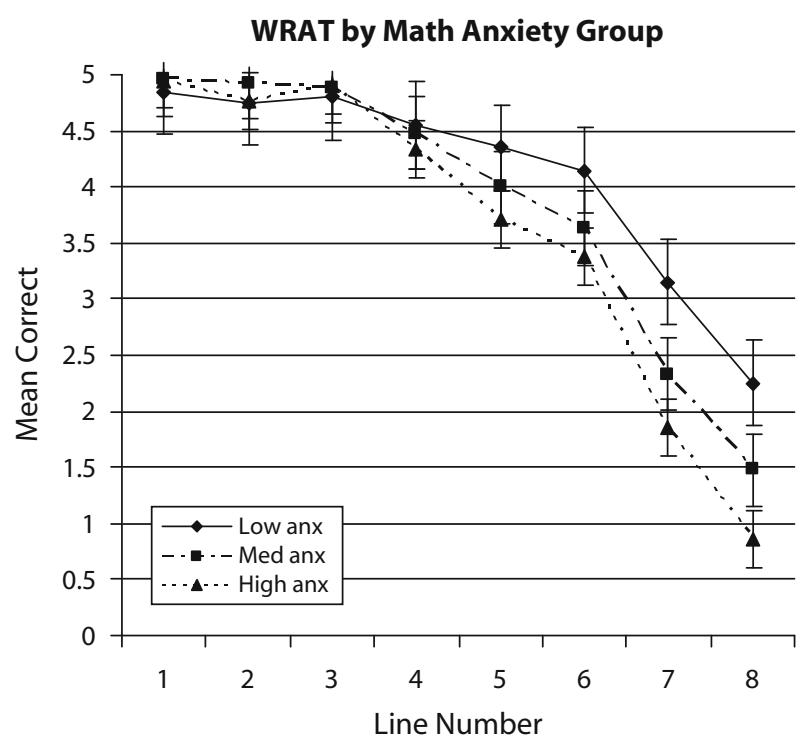

Figure 2. Mean number correct per line (out of five) on the Wide Range Achievement Test (WRAT) for low-, medium-, and high-math-anxious groups. 
ment and math anxiety. Disentangling that confound at higher levels of difficulty will be much more difficult.

\section{Working Memory and Math Anxiety}

The question of how math anxiety compromises working memory has a more subtle answer than merely saying that math anxiety consumes the resources of the working memory system. For example, we used two different verbal-based span assessments, and found no significant anxiety-group differences at all. But when a computationbased span task was administered, we found a pronounced decline in assessed working memory capacity; the fullscale correlation was a significant - -40 (Ashcraft \& Kirk, 2001, Experiments 1 and 3). We argue that a math-anxious person's working memory resources are drained - that the individual suffers a compromised working memory — only when the actual math anxiety is aroused, as in span tasks that involve computations.

To demonstrate the joint effects of working memory and math anxiety, we had our participants do two-column addition, either alone or in combination with a letter-recall secondary task (Ashcraft \& Kirk, 2001, Experiment 2). As Figure 3 shows, errors to the letter task grew only modestly for the low-, medium-, and high-anxiety groups in the control conditions and in the two-letter load condition. But in the difficult six-letter condition, with the workingmemory-demanding carry problems, the effect of the dual task was quite strong, and affected the high-anxious group the most. Clearly, when the math task becomes demanding, and when the necessary resources from working memory are occupied by the secondary task, performance suffers. Highly anxious participants, who are already wasting working memory resources by attending to their own anxiety, suffer the most (if the dual task per se induced, say, high-state anxiety, independent of working memory, then errors should have increased on all dual task trials, including the noncarry problems). We are currently exploring other ways in which this diversion of working memory affects the outcomes of mental processing.

A related anxiety effect bears brief mention as well. Our results show that high-math-anxious participants often sacrifice accuracy for speed, especially as problems become more difficult, which we interpreted as an avoidance-like effort to finish the testing session as quickly as possible (Faust et al., 1996). Consequences of this - say, in terms of achievement testing or learning from homework-have yet to be investigated.

\section{Applications to Education}

Math is an important topic in schooling and in preparation for careers; skill at math is often a filter in terms of career pathways. Math is also a cognitively challenging topic, one that involves manipulation of symbols in an often highly abstract setting. Furthermore, math must be taught in school, unlike language, which children learn naturally from their surroundings early in life. Presumably, this should give cognitive psychology some advantages in studying math, given that points in the math curriculum can be specified - for example, the grade level at which prealgebra is introduced.
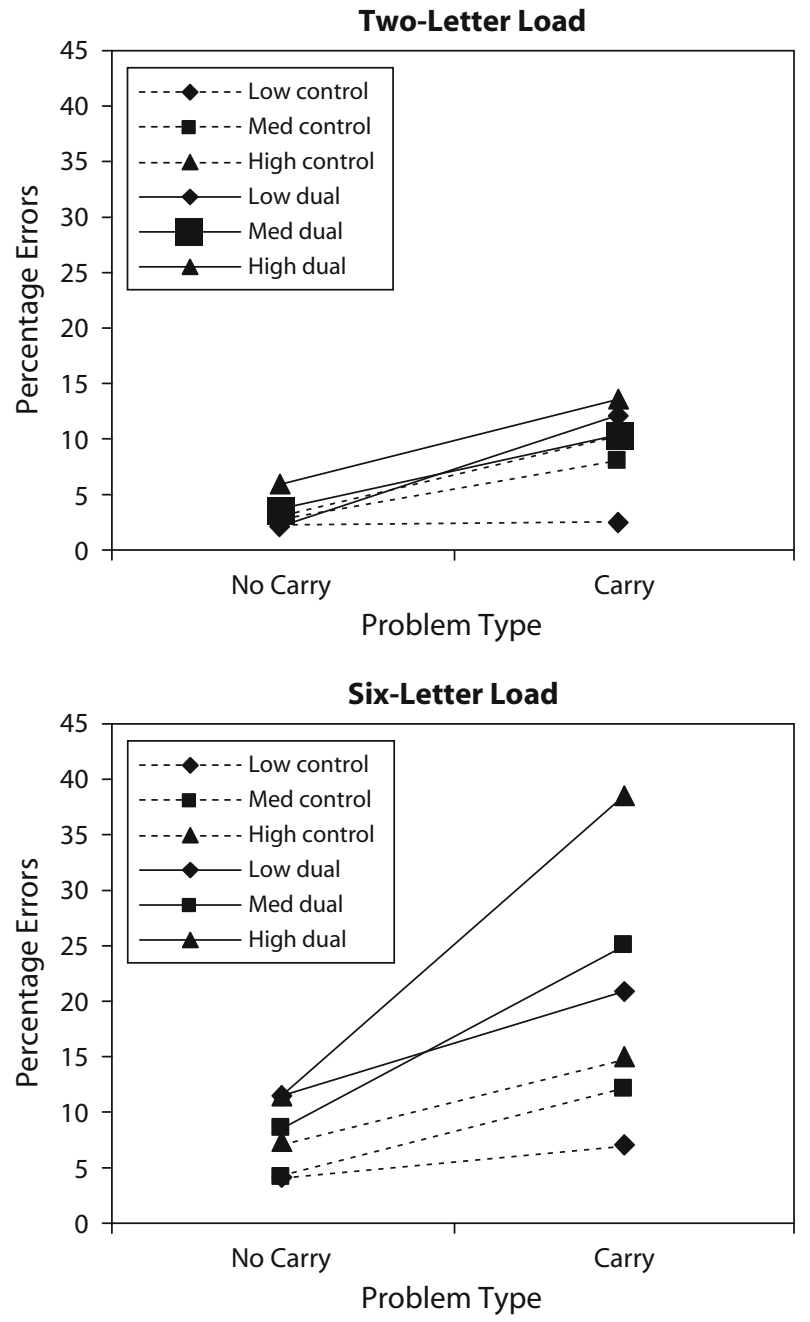

Figure 3. Percentage errors to the letter task in control and dual task conditions, separately for two- versus six-letter memory loads and low-, medium-, and high-math-anxious groups. From "The relationships among working memory, math anxiety, and performance," by M. H. Ashcraft \& E. P. Kirk, 2001, Journal of Experimental Psychology: General, 130, p. 231, Figure 2. Copyright 2001 American Psychological Association. Adapted with permission.

It bears repeating that there is a pervasive reliance throughout arithmetic and math on the working memory system, from simple counting and estimation processes (see, e.g., Siegler \& Booth, 2005) up through algebra and complex problem solving (Ayres, 2001). Indeed, even at the earliest levels of formal education, there is a strong relationship between a child's working memory span and performance on number-based tasks (see, e.g., Adams $\&$ Hitch, 1997). The need for working memory may be easy to overlook, given that even a difficult mathematics problem can be presented with far fewer symbols than, say, a complex, multiclause sentence. It is also the case that sentences, and the words that compose them, generally refer to more concrete concepts and ideas (objects, actions, events) than a typical math problem - even a word problem in math-does. The very abstractness of 
mathematical symbols surely adds to the difficulties that people encounter when learning math, including difficulties in storing and using information in working memory. Acquiring the capacity for abstract thinking, of course, is a late developmental milestone. So far, relatively few projects have explored math processing beyond the four basic arithmetic operations, so the role of working memory at higher levels of math has hardly been investigated at all. Based on the central role identified so far, however, it can only be the case that more difficult math will be even more dependent on working memory. This would be especially true given the heavier burden on procedural processing at higher levels of math, and the lesser degree of automaticity that might be attained by those procedures (the degree to which math procedures themselves can become more automatic is an almost totally ignored topic).

Turning now to math anxiety, several implications for education can be drawn. Math anxiety seems to influence cognitive processing in a straightforward way-working memory resources are compromised whenever the anxiety is aroused. Given the pervasiveness of working-memorydependent processing in arithmetic and math, this predicts serious effects of math anxiety. It is easy to imagine how math anxiety affects learning - say, in a high school math classroom. A student whose math anxiety is aroused is diverting needed attention away from the content of the class and toward internal worries and anxieties over math. This can only slow or degrade the mastery of the to-belearned information.

Further, the implications in the correlational literature seem unavoidable. Math anxiety leads to a global avoidance pattern - whenever possible, students avoid taking math classes and avoid situations in which math will be necessary, including career paths. In an important study of math teachers' approaches to teaching, Turner et al. (2002) showed how students with an unsupportive, "cold" teacher avoid in-school behaviors (making eye contact with the teacher, going to out-of-class help sessions). These sound like the ingredients for math anxiety. We predict that math anxiety is learned in the classroom-for example, when a student is called to the board to work a problem, does poorly, and is embarrassed in front of the teacher and his or her peers. In short, lower-than-average math abilities and/or working memory capacity, susceptibility to public embarrassment, and a nonsupportive teacher all may be risk factors for developing math anxiety (Ashcraft et al., 2007). Once math anxiety is established, it then seems to be supported by a variety of cultural attitudes that undermine math achievement-for example, that math is hard, one either is or is not good at math, regardless of how hard one works, and so on.

We speculate one step further on the teacher's role in the development of math anxiety. When college majors are given a math anxiety test, those who average the highest are individuals preparing to be elementary school teachers (Hembree, 1990). Compounding this, students earning such degrees are typically required to take very few math courses (see also Ma, 1999). We thus suggest that the stage is set early on in math education for students to be "stranded" without a reasonable, instructive expla- nation for many aspects of math, and/or in a classroom in which the teacher, possibly defensively, adopts an unsupportive, "cold" teaching approach. Placing an at-risk child into such a teacher's class may be the ideal recipe for creating math anxiety, a hypothesis we are beginning to investigate.

Cognitive psychology's role should be to examine the acquisition and mastery of math and math procedures, and also the ways in which math anxiety has consequences for cognitive processing. Cognition can also help determine the developmental course of this learned anxiety, and explore the possibility that some genuinely cognitive factors - for example, low math aptitude or working memory capacity - may be risk factors for math anxiety. Investigating the cognitive consequences of math anxiety may provide a rather unique opportunity, testing the impact of a malleable individual difference, math anxiety, and its consequences on cognitive processing.

\section{AUTHOR NOTE}

Correspondence concerning this article should be addressed to M. H. Ashcraft, Psychology Department, University of Nevada, Las Vegas, Box 455030, 4505 South Maryland Parkway, Las Vegas, NV 891545030 (e-mail: mark.ashcraft@unlv.edu).

\section{REFERENCES}

Adams, J. W., \& Hitch, G. J. (1997). Working memory and children's mental addition. Journal of Experimental Child Psychology, 67, 21-38.

AshCraft, M. H., \& KIRK, E. P. (2001). The relationships among working memory, math anxiety, and performance. Journal of Experimental Psychology: General, 130, 224-237.

AshCraft, M. H., Krause, J. A., \& HopKo, D. R. (2007). Is math anxiety a mathematical learning disability? In D. B. Berch \& M. M. M. Mazzocco (Eds.), Why is math so hard for some children? The nature and origins of mathematical learning difficulties and disabilities (pp. 329-348). Baltimore: Brookes.

AshCRAFT, M. H., \& RidLey, K. S. (2005). Math anxiety and its cognitive consequences: A tutorial review. In J. I. D. Campbell (Ed.), Handbook of mathematical cognition (pp. 315-327). New York: Psychology Press.

AYres, P. L. (2001). Systematic mathematical errors and cognitive load. Contemporary Educational Psychology, 26, 227-248.

Beilock, S. L., Kulp, C. A., Holt, L. E., \& CarR, T. H. (2004). More on the fragility of performance: Choking under pressure in mathematical problem solving. Journal of Experimental Psychology: General, 133, 584-600.

CAMPBell, J. I. D. (ED.) (2005). Handbook of mathematical cognition. New York: Psychology Press.

Campbell, J. I. D., \& Charness, N. (1990). Age-related declines in working memory skills: Evidence from a complex calculation task. Developmental Psychology, 26, 879-888.

CAmpbell, J. I. D., \& Xue, Q. (2001). Cognitive arithmetic across cultures. Journal of Experimental Psychology: General, 130, 299-315.

Faust, M. W., Ashcraft, M. H., \& Fleck, D. E. (1996). Mathematics anxiety effects in simple and complex addition. Mathematical Cognition, 2, 25-62.

FÜrst, A. J., \& Hıтch, G. J. (2000). Separate roles for executive and phonological components of working memory in mental arithmetic. Memory \& Cognition, 28, 774-782.

HamanN, M. S., \& AshCRAFT, M. H. (1986). Textbook presentations of the basic addition facts. Cognition \& Instruction, 3, 173-192.

HeCHT, S. A. (2002). Counting on working memory in simple arithmetic when counting is used for problem solving. Memory \& Cognition, 30, 447-455.

Hembree, R. (1990). The nature, effects, and relief of mathematics anxiety. Journal for Research in Mathematics Education, 21, 33-46. 
LeFevre, J.-A., DeStefano, D., Coleman, B., \& Shanahan, T. (2005). Mathematical cognition and working memory. In J. I. D. Campbell (Ed.), Handbook of mathematical cognition (pp. 361-378). New York: Psychology Press.

LeFevre, J.-A., SAdesky, G. S., \& Bisanz, J. (1996). Selection of procedures in mental addition: Reassessing the problem-size effect in adults. Journal of Experimental Psychology: Learning, Memory, \& Cognition, 22, 216-230.

Logie, R. H., Gilhooly, K. J., \& Wynn, V. (1994). Counting on working memory in arithmetic problem solving. Memory \& Cognition, 22, 395-410.

MA, L. (1999). Knowing and teaching elementary mathematics: Teachers' understanding of fundamental mathematics in China and the United States. Mahwah, NJ: Erlbaum.

Seyler, D. J., KirK, E. P., \& AshCraft, M. H. (2003). Elementary subtraction. Journal of Experimental Psychology: Learning, Memory, \& Cognition, 29, 1339-1352.
Siegler, R. S., \& Booth, J. L. (2005). Development of numerical estimation. In J. I. D. Campbell (Ed.), Handbook of mathematical cognition (pp. 197-212). New York: Psychology Press.

Siegler, R. S., \& Shrager, J. (1984). A model of strategy choice. In C. Sophian (Ed.), Origins of cognitive skills (pp. 229-293). Hillsdale, NJ: Erlbaum.

Tronsky, L. N. (2005). Strategy use, the development of automaticity, and working memory involvement in complex multiplication. Memory \& Cognition, 33, 927-940.

Turner, J. C., Midgley, C., Meyer, D. K., Gheen, M., Anderman, E. M., Kang, Y., \& PATrick, H. (2002). The classroom environment and students' reports of avoidance strategies in mathematics: A multimethod study. Journal of Educational Psychology, 94, 88-106.

Zbrodoff, N. J., \& Logan, G. D. (2005). What everyone finds: The problem-size effect. In J. I. D. Campbell (Ed.), Handbook of mathematical cognition (pp. 331-345). New York: Psychology Press. 